\title{
The timing of tuberculosis after isoniazid preventive therapy among gold miners in South Africa: a prospective cohort study
}

Sabine M. Hermans ${ }^{1,2,3,4^{*}}$, Alison D. Grant ${ }^{1,5,6}$, Violet Chihota ${ }^{5,7}$, James J. Lewis ${ }^{1}$, Emilia Vynnycky ${ }^{1,8}$, Gavin J. Churchyard ${ }^{1,5,7,9}$ and Katherine L. Fielding ${ }^{1,5}$

\begin{abstract}
Background: The durability of isoniazid preventive therapy (IPT) in preventing tuberculosis (TB) is limited in highprevalence settings. The underlying mechanism (reactivation of persistent latent TB or reinfection) is not known. We aimed to investigate the timing of TB incidence during and after IPT and associated risk factors in a very high TB and HIV-prevalence setting, and to compare the observed rate with a modelled estimate of TB incidence rate after IPT due to reinfection.

Methods: In a post-hoc analysis of a cluster-randomized trial of community-wide IPT among South African gold miners, all intervention arm participants that were dispensed IPT for at least one of the intended 9 months were included. An incident TB case was defined as any participant with a positive sputum smear or culture, or with a clinical TB diagnosis assigned by a senior study clinician. Crude TB incidence rates were calculated during and after IPT, overall and by follow-up time. HIV status was not available. Multivariable Cox regression was used to analyse risk factors by follow-up time after IPT. Estimates from a published mathematical model of trial data were used to calculate the average reinfection TB incidence in the first year after IPT.
\end{abstract}

Results: Among 18,520 participants (96\% male, mean age 41 years, median follow-up 2.1 years), 708 developed TB. The TB incidence rate during the intended IPT period was 1.3/100 person-years (pyrs; $95 \%$ confidence interval (Cl), 1.0-1.6) and afterwards $2.3 / 100$ pyrs ( $95 \% \mathrm{Cl}$, 1.9-2.7). TB incidence increased within 6 months followed by a stable rate over time. There was no evidence for changing risk factors for TB disease over time after miners stopped IPT. The average TB incidence rate attributable to reinfection in the first year was estimated at 1.3/100 pyrs, compared to an observed rate of $2.2 / 100$ pyrs (95 \% Cl, 1.8-2.7).

Conclusions: The durability of protection by IPT was lost within 6-12 months in this setting with a high HIV prevalence and a high annual risk of $M$. tuberculosis infection. The observed rate was higher than the modelled rate, suggesting that reactivation of persistent latent infection played a role in the rapid return to baseline TB incidence.

Keywords: Isoniazid preventive therapy, Latent infection, Reactivation, Reinfection, Tuberculosis

\footnotetext{
*Correspondence: s.hermans@aighd.org

${ }^{1}$ TB Centre, London School of Hygiene \& Tropical Medicine, London, UK

${ }^{2}$ Department of Global Health, Academic Medical Center, University of

Amsterdam, Amsterdam Institute for Global Health and Development,

Amsterdam, The Netherlands

Full list of author information is available at the end of the article
} 


\section{Background}

The world has seen an upsurge in tuberculosis (TB) incidence since the advent of the human immunodeficiency virus (HIV) epidemic in the 1990s. This rise was mainly seen in the sub-Saharan African region, which accounts for $74 \%$ of the annual 1.2 million people living with HIV who develop TB [1]. In southern Africa, HIV prevalence among people with TB disease is around $50 \%$.

Isoniazid preventive therapy (IPT) is part of the World Health Organization (WHO)'s 3Is strategy formulated to reduce the burden of TB in people living with HIV [2]. A 6-12 month course of daily isoniazid has been shown to reduce TB incidence in those with a positive tuberculin skin test (TST) by an average of $60 \%$ [3]. In a recent randomised controlled trial of IPT in patients on antiretroviral therapy (ART), those with a negative TST at enrolment also benefitted [4], although these participants could have undergone TST conversion whilst on ART [5]. A large cluster-randomised trial of communitywide IPT among 78,744 gold mine workers in South Africa, the Thibela TB study [6], did not find a protective effect of IPT on TB incidence or TB prevalence at a population level. At an individual level, the direct protective effect of IPT was calculated in the subset of the study population included in the baseline prevalence survey, which was a random sample of the total workforce. This showed a $58 \%$ (95\% confidence interval (CI), $12-80 \%)$ lower incidence rate of TB during the 9 months on IPT versus that in a control cohort. However, this protective effect disappeared within the first 9 months after the intended IPT period.

Other studies have also shown that the effect of IPT wanes over time in settings with high annual risks of Mycobacterium tuberculosis infection [4, 7-9]. Few IPT trials have reported data on long-term TB incidence rates after IPT discontinuation, and on how these rates change over time. Additional file 1: Table S1 gives an overview of long-term follow-up data of the adult trials. The Bethel studies in the pre-HIV era showed a durable effect over a long period of time, but were performed in a context of reducing $\mathrm{TB}$ transmission and other $\mathrm{TB}$ control strategies $[10,11]$. In contrast, the studies performed in TB high-prevalence settings showed a rapid increase in TB incidence rates after IPT cessation. The underlying mechanism (reactivation of persistent latent infection or reinfection) is not well understood.

The relatively small numbers of events in these cohorts have limited the power to investigate the timing of TB occurring after IPT cessation and the change of associated risk factors over time. We therefore set out to analyse TB incidence in the entire Thibela TB study population that had received IPT. This population was almost four times as large as the IPT cohort used to calculate the direct effect of the intervention, which were the participants included in the baseline survey only (a random sample of 1,000 miners per cluster on whom more in-depth data was collected) [6].

Our study objectives were four-fold: to estimate the $\mathrm{TB}$ incidence rate during and after IPT (objective 1); to investigate risk factors associated with incident TB after IPT (objective 2); and to determine whether these differed over time after the end of IPT (effect modification by follow-up period, objective 3 ). All three analyses used both the intended and actual duration of IPT (Fig. 1). Our final objective was to compare the observed incidence of TB in the first year after IPT with a crude estimate of the average incidence rate of TB disease due to reinfection that might have occurred during that period (objective 4).

\section{Methods}

\section{Study design and setting: Thibela TB trial}

This was a post-hoc analysis of data from the Thibela TB study, a cluster-randomised trial to investigate the impact of community-wide IPT on TB incidence and prevalence compared to standard of care among 15 clusters (mine shafts) of gold mine workers in South Africa [6]. Clusters were randomised to the intervention (TB screening offered to the entire cluster linked to treatment for TB disease or infection, as appropriate; eight clusters) or control (standard of care, seven clusters)

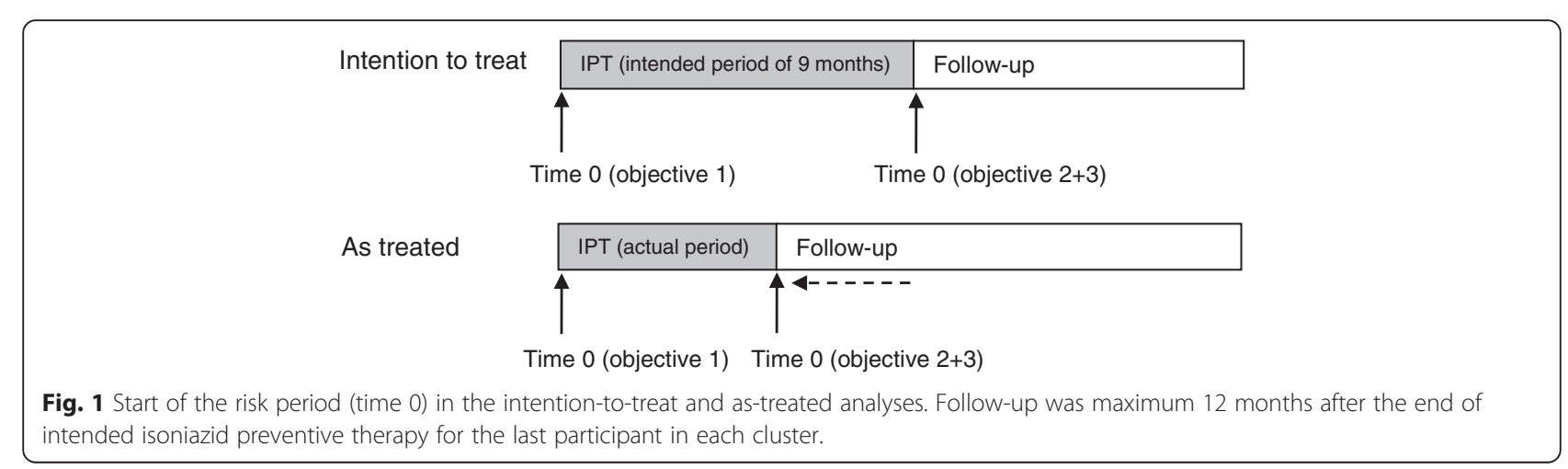


arm [12]. The study methods are described in full elsewhere [12].

This analysis focused solely on the eight intervention clusters. Within each cluster, the same procedure was followed for enrolment and follow-up (Additional file 1: Figure S1). All mine workers within the cluster were invited to participate [13]. As $89 \%$ of the workforce was estimated to be infected with $M$. tuberculosis, TST was deemed unnecessary [14]. Consenting miners were screened for TB using a symptom screen and a chest Xray; a sputum smear and culture was ordered for miners with signs or symptoms suggestive of TB. Positive laboratory results and/or high clinical suspicion were reason for referral to the mine health services for further investigation and, if necessary, TB treatment. Participants without evidence of active TB or contraindications to IPT were offered 9 months of IPT (300 mg isoniazid daily) [15]. During IPT, participants were seen monthly by study staff for dispensing of study medication, and a symptom screen for TB and adverse events. Symptomatic patients were referred to the mine health services for further work-up and management. After cessation of IPT, participants were evaluated for incident TB during 6-monthly or annual routine chest $\mathrm{X}$-ray screening by occupational health services or after self-presentation with symptoms to the mine health services.

\section{Population included in post-hoc analysis}

All participants in the eight intervention clusters who were prescribed IPT at least once and who were permanent employees were included in this analysis. For analyses involving follow-up after the end of IPT, only participants who had completed the IPT period without a TB diagnosis, alive and still in the workforce, were included. In each cluster, follow-up was continued until 1 year after the end of the last participant's intended IPT period.

\section{Definitions and measurement of variables}

Baseline characteristics of study participants were collected by a questionnaire at enrolment into the intervention and prior to initiation of IPT. At the request of the trade unions, no HIV testing was performed for study purposes. However, use of ART as concomitant medication was asked with respect to assessing possible adverse events. HIV prevalence among miners was estimated at $29 \%$ in 2000 [16]. IPT dispensing dates were recorded; the number of monthly refill visits was used as a proxy of adherence ( $\geq 6$ defined as optimal, 6 months being a recommended duration for IPT at the time the trial was designed [17]). The end of the intended IPT period was defined as 270 days from the first prescription date and the end of the actual IPT period as 30 days from the last dispensing date.
For estimation of the TB incidence rate, the start of the risk period was defined as the date of IPT initiation (objective 1); for the risk factor analyses the risk period started at the end of the intended or actual IPT period (objectives 2 and 3).

Miners with symptoms or signs suggesting TB were investigated and treated by the mine health services. Data on these episodes were obtained from record review. Routine diagnostic workup included sputum smear microscopy and a chest X-ray. Only one mining company routinely used mycobacterial culture to evaluate all miners with signs/symptoms suggestive of $\mathrm{TB}$, the others only for those with a history of prior TB treatment. However, during the study, we endeavoured, following additional consent, to collect an additional sputum sample for smear microscopy and culture, though full coverage of this was not achieved. Cultures showing only non-tuberculous mycobacteria were not considered as TB. TB diagnoses were categorised as definite ( 2 positive smears or 1 positive culture for $M$. tuberculosis, or histological evidence of TB at autopsy in TB cases only ascertained post-mortem), probable (1 positive smear or culture with unidentified mycobacteria) or possible (clinical or radiological signs and symptoms, but no or negative smear/culture results). Medical records of participants with possible TB were reviewed by senior study clinicians, masked to study arm, to arbitrate on whether to include as incident TB.

Employment records were used to determine dates of employment and reasons for leaving the workforce (including deaths). Miners who died and underwent autopsy which revealed histological evidence of $\mathrm{TB}$ were included as incident TB cases.

The end of the risk period was defined as the earliest of TB treatment initiation (irrespective of the method of diagnosis), termination from the workforce, death or the end of follow-up. Participants who left the workforce due to being 'medically boarded' (discontinuing employment for a medical reason) were all evaluated by the mine healthcare system for active TB prior to the end of employment, and we therefore assumed that none of these represented missed TB diagnoses.

\section{Statistical methods}

The primary analyses were carried out using the intended IPT period to define the end of IPT (intentionto-treat (ITT), Fig. 1). Three sensitivity analyses were conducted: an as-treated (AT) analysis using the actual end date of IPT (Fig. 1), an AT analysis restricted to those with optimal adherence (defined as having been dispensed $\geq 6$ months of IPT, see below), and a repeat analysis of both the ITT and AT analyses with a stricter $\mathrm{TB}$ case definition restricted to definite and probable TB diagnoses. 
We calculated the overall TB incidence rate after initiation of IPT and period-specific rates during and after IPT. Poisson regression analyses adjusted for clustering by mine shaft were used to calculate TB incidence rates and to determine the rate ratio for $\mathrm{TB}$ after stopping IPT compared to during IPT. We investigated trends in TB incidence rates over time after the end of IPT using tests for linearity and departure from linearity.

Multivariable Cox regression analysis was used to investigate risk factors for TB following the end of IPT. We included factors we considered a priori as risk factors for TB (sex, age, previous TB, number of years in the workforce, type of work (reduced ventilation with underground work), housing (crowding in hostels), selfreported use of ART and number of times IPT was dispensed). The potential confounder (country of origin) was retained in the model if inclusion led to a change in hazard ratio estimate. In case of co-linearity, the more important of the co-linear variables was determined by literature review and retained. We considered previous TB might be on the causal pathway as it has been shown to be a risk factor for recurrent TB $[18,19]$. We therefore examined the effect of dropping this variable in the final multivariable Cox model. The effect of excluding self-reported ART use was also examined. The final model was adjusted for calendar time.

Clustering was controlled for with a fixed effect for cluster in all regression analyses. Random effects analysis was not used due to small number of clusters [20]. Cluster estimates were not reported.

Departure from the proportional hazards assumption was tested in objective 3: we incorporated interaction terms between the time period of follow-up and the a priori risk factors. To maximise power, we used unadjusted analyses and two time periods: $<12$ and $\geq 12$ months after stopping IPT. This time split was chosen to correspond with the expected timing of different underlying mechanisms (reactivation of inadequately treated latent infection $<12$ months versus reinfection $\geq 12$ months), extrapolated from a study of recurrent disease after treatment [21].

For the AT analysis, these procedures were repeated using the actual end date of IPT as defined above. Twosided $P$ values in regression analyses were derived from likelihood ratio tests. Departure from linearity was tested for all ordered categorical variables. The analyses were conducted using STATA version SE 12.1 (College Station, Texas, USA).

\section{Comparison between observed and modelled TB incidence in the first year after IPT}

We applied base-case assumptions used in the published mathematical model of the Thibela study data to calculate a crude estimate of the average incidence rate of TB disease attributable to reinfection in the first year after IPT [22]. We did this overall and by cluster to allow for the varying annual risk of infection per cluster. We thereby assumed an annual risk of infection (averaged across clusters) before the intervention of $20 \%$ per year and, based on outputs from the original model [22], a reduction in the annual risk of infection ranging between 11-20\% after IPT was introduced. For all clusters we assumed an HIV prevalence of $30 \%$ of whom $25 \%$ had a CD4 count below 200 cells/ $\mu \mathrm{L}$ (and all of whom were eligible for and on ART), an initial pre-treatment loss to follow-up of $40 \%$ and an average time to detection of 1 year. The age distribution and prevalence of silicosis depended on the cluster and were based on data collected as part of the study. The origin of these assumptions are explained in detail elsewhere [22]. Furthermore, also based on the original model, we assumed that reinfection, but no progression, could take place during IPT. We then calculated the observed TB incidence rate in the first 12 months after IPT (ITT), overall and per cluster, and compared these rates to the modelled estimates of TB incidence.

\section{Ethical review}

The Research Ethics Committees of University of KwaZulu-Natal, South Africa, and the London School of Hygiene \& Tropical Medicine (LSHTM) as well as the South African Medicines Control Council and the South African Safety in Mines Research Advisory Committee gave approval for the Thibela TB study. The LSHTM ethics committee gave approval for this post-hoc secondary data analysis. Miners who participated in the intervention provided written or witnessed oral informed consent.

\section{Results}

Between July 2006 and February 2010, 18,520 participants were prescribed IPT at least once and were permanent employees. Their baseline characteristics are described in Table 1; $96 \%$ were men, in keeping with the demographics of this workforce, with a median age of 41 years and a median time in the mining workforce of 18 years. Overall, $57 \%$ of mine workers were South African, $91 \%$ worked mainly underground, $59 \%$ lived in a hostel, and $12 \%$ reported having had TB previously and $2.7 \%$ as being on ART. IPT was dispensed six or more times to 11,293 (61 \%) participants, three to five times to $2,125(12 \%)$, and once or twice to $5,102(28 \%)$ participants.

\section{TB incidence rate during and after IPT}

Across all clusters, 708 participants developed TB during 37,321 person-years (pyrs). Of these, $55 \%$ were definite, $12 \%$ probable and $33 \%$ possible diagnoses; 541 were 
Table 1 Baseline characteristics of the study population $(n=18,520)$

\begin{tabular}{lll}
\hline Characteristic & & Total, $\mathrm{n}(\mathrm{col} \%)^{\mathrm{a}}$ \\
\hline Sex & Male & $17,763(95.9)$ \\
Age, years & $\leq 29$ & $2,456(13.3)$ \\
& $30-39$ & $4,919(26.6)$ \\
& $40-49$ & $7,782(42.0)$ \\
& $\geq 50$ & $3,362(18.2)$ \\
Country of origin & South Africa & $10,501(56.7)$ \\
& Lesotho & $5,178(28.0)$ \\
& Mozambique & $1,854(10.0)$ \\
Years in workforce & Other & $979(5.3)$ \\
& $0-9$ & $4,874(26.4)$ \\
Type of work & $10-19$ & $5,017(27.2)$ \\
Type of housing & $20-29$ & $5,846(31.7)$ \\
Previous TB b & $\geq 30$ & $2,724(14.8)$ \\
Previous IPT & Underground & $16,821(91.2)$ \\
Self-reported ART use & Hostel & $10,913(58.9)$ \\
a & & $2,212(12.0)$ \\
& & $79(0.4)$ \\
& & $521(2.8)$
\end{tabular}

${ }^{a}$ Missing data (n): sex (1), age (46), country of origin (8), years in workforce (59), type of work (77), type of residence (1), previous TB (14), previous IPT (38), self-reported ART use (20)

${ }^{\mathrm{b}}$ Timing of previous TB episodes: median 4.0 years (IQR, 1.8-8.9 years), 5 had missing TB dates; 265 (12\%) episodes occurred in year prior to IPT ART, Antiretroviral therapy; IPT, Isoniazid preventive therapy; IQR, Interquartile range; $\mathrm{N}$, Number; $\mathrm{TB}$, Tuberculosis diagnosed after the intended end of IPT (ITT analysis) and 638 after the actual end of IPT (AT analysis, Fig. 2). The median follow-up time, including the IPT period, was 2.1 years and varied by cluster (ranging between 1.9 and 2.5 years).

In the ITT analysis, the overall $\mathrm{TB}$ incidence rate on IPT was 1.3/100 pyrs (95\% CI, 1.0-1.6), whereas after IPT it was 2.3 (95\% CI, 1.9-2.7) with a rate ratio (RR) of 1.8 (95\% CI, 1.4-2.3). In the AT analysis, the rate on IPT was $0.7 / 100$ pyrs (95\% CI, 0.5-1.0), whereas after IPT it was $2.3 / 100$ pyrs (95 \% CI, 1.9-2.7) with a RR of 3.1 (95 \% CI, 2.3-4.3). Among those in the AT analysis who took 6 months or more of IPT, the incidence rate on IPT was $0.3 / 100$ pyrs (95\% CI, 0.2-0.4) and off IPT $2.2 / 100$ pyrs $(95 \% \mathrm{CI}, 1.8-2.7)$, with a RR of $6.7(95 \%$ CI, 5.6-8.0).

After the end of IPT, from the test for linearity, there was some evidence for an increasing rate over time in the ITT analysis and in the subgroup who took 6 months or more of IPT, but not in the AT analysis (Fig. 3). The results of the sensitivity analyses excluding possible $\mathrm{TB}$ diagnoses were similar (Additional file 1: Figure S2). As expected, the overall TB incidence rates were lower, but the overall pattern of changes of TB incidence over time was similar.

\section{Risk factors associated with incident TB after IPT}

A total of 17,446 (94\%) participants completed the intended IPT period of 9 months alive, in the workforce and not taking TB treatment, and formed the cohort for the risk factor analysis for incident TB after IPT (Fig. 2). They contributed 24,037 person-years, a median followup of 1.4 years. Neither their baseline characteristics nor the proportion of participants who took 6 months or

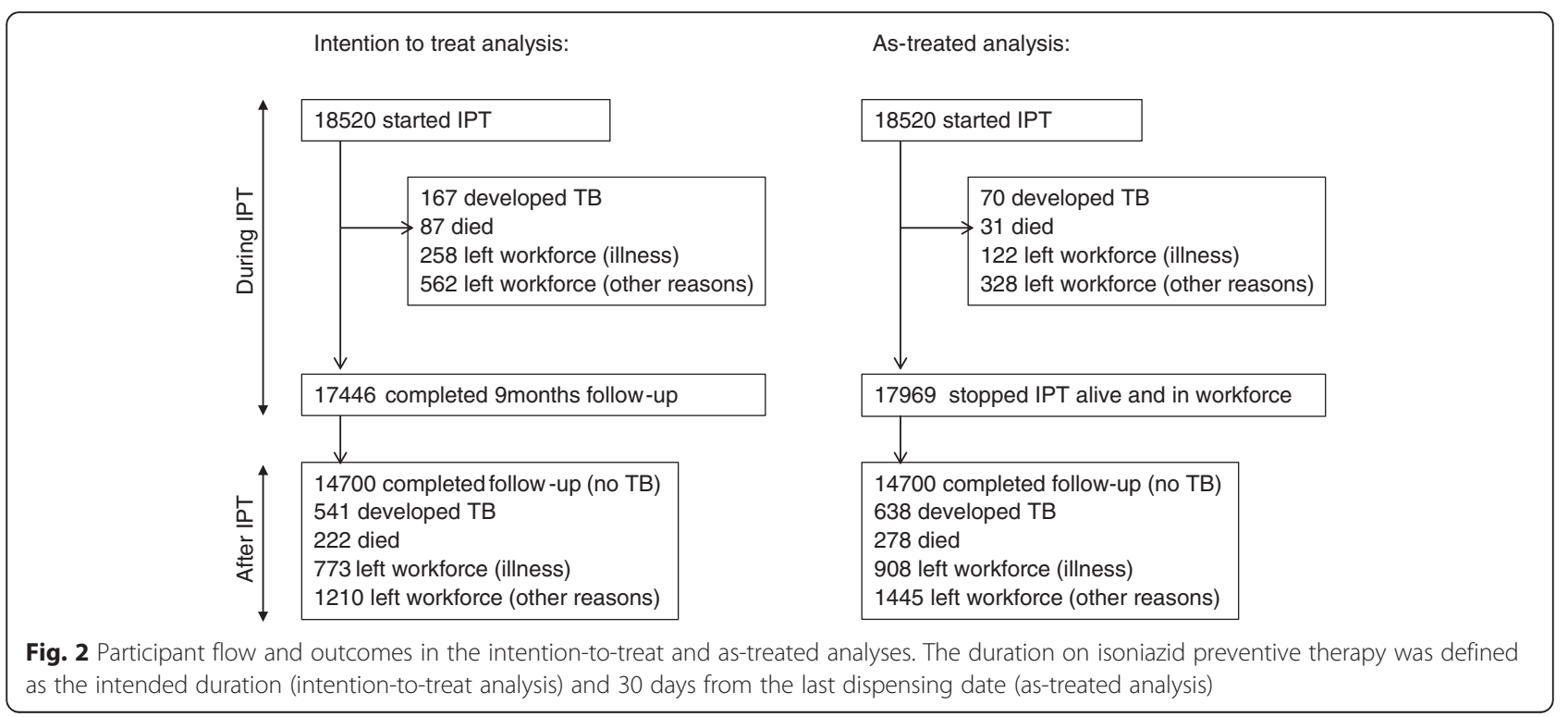




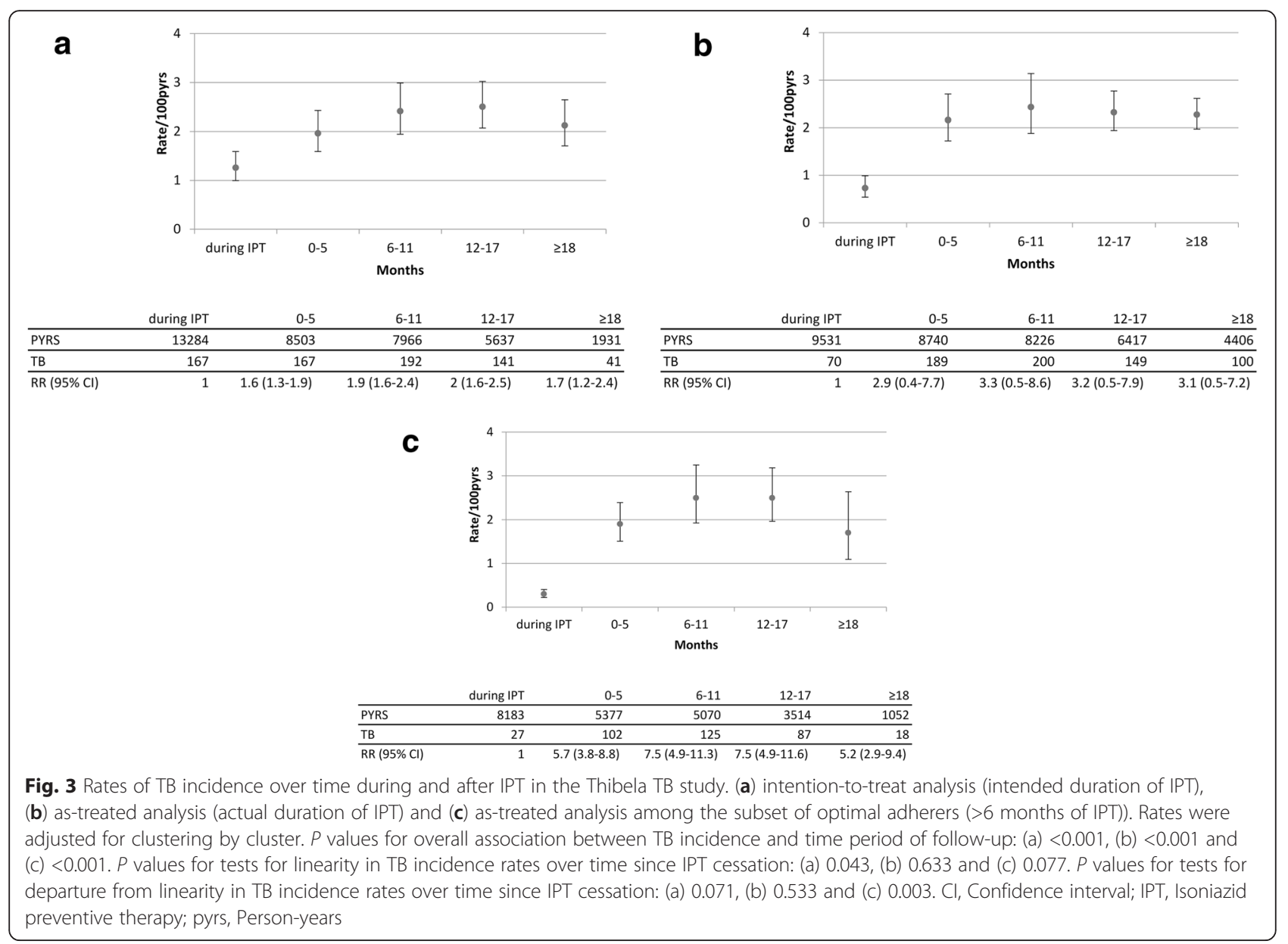

more of IPT differed from the original cohort (Additional file 1: Table S2). Characteristics of participants leaving the workforce for reasons other than ill health were compared to those of all participants. On average, these participants were older, had been in the workforce longer and had had TB previously (Additional file 1: Table S3).

After adjusting for sex, country of origin, self-reported ART use and duration of IPT taken, there was strong evidence of an increased hazard of TB following IPT among participants who were older, doing underground rather than surface work or had had TB before (Table 2). A decreased hazard of TB was found in those not living in a hostel or who had more IPT dispensed. Age and years in the workforce were found to be co-linear; we retained age in the model as this had been previously shown to be the stronger risk factor for TB [23]. Excluding previous $\mathrm{TB}$ or self-reported ART use from the model did not change the results, nor did adjustment for calendar time.

The risk factor analysis in the AT cohort comprised 16,962 pyrs in the first year after IPT and 10,822 pyrs from the second year onwards. The risk factor estimates were very similar to the ITT analysis (Additional file 1: Table S3).

\section{Effect modification of risk factors by follow-up period}

No evidence for effect modification of risk factors by follow-up period was found; associated risk factors were similar in the first year of follow-up compared to afterwards (Table 3). This included the effect of number of doses of IPT by follow-up time period. The results of this analysis in the AT cohort were very similar (Additional file 1: Table S4).

\section{Comparison between the observed and estimated TB incidence rate after IPT}

The observed TB incidence rate during the first year after IPT (ITT analysis) was $2.18 / 100$ pyrs $(95 \% \mathrm{CI}$, 1.79-2.66). The crude average incidence rate of TB disease due to reinfection that might have occurred in the first year after IPT was estimated at 1.28 per 100 pyrs. At a cluster level, the estimated rates were consistently lower than the rates observed in the data (Additional file 1: Table S6). 
Table 2 Risk factors for TB incidence after IPT (intention-to-treat)

\begin{tabular}{|c|c|c|c|c|c|c|c|c|}
\hline Total & & $\mathrm{n}(\%)$ & pyrs & TB & $\mathrm{HR}(95 \% \mathrm{Cl})^{a}$ & $P$ value & $\mathrm{aHR}(95 \% \mathrm{Cl})^{\mathrm{a}, \mathrm{f}}$ & $P$ value \\
\hline \multirow[t]{2}{*}{ Sex } & Male & 16,704 (95.8) & 23,036 & 523 & 1 & 0.08 & 1 & 0.73 \\
\hline & Female & 741 (4.2) & 999 & 18 & $0.67(0.42-1.08)$ & & $1.09(0.67-1.80)$ & \\
\hline \multirow[t]{4}{*}{ Age, years ${ }^{b}$} & $\leq 29$ & 2,370 (13.6) & 3,221 & 30 & 1 & $<0.001$ & 1 & $<0.001$ \\
\hline & $30-39$ & 4,677 (26.8) & 6,660 & 131 & $2.13(1.43-3.17)$ & & $2.18(1.46-3.25)$ & \\
\hline & $40-49$ & $7,363(42.2)$ & 10,291 & 276 & $2.81(1.92-4.10)$ & & $2.84(1.92-4.18)$ & \\
\hline & $\geq 50$ & 3,035 (17.4) & 3,863 & 104 & $2.69(1.79-4.06)$ & & $2.77(1.81-4.23)$ & \\
\hline \multirow[t]{4}{*}{ Country of origin } & South Africa & $10,008(57.4)$ & 13,729 & 284 & 1 & 0.001 & 1 & 0.09 \\
\hline & Lesotho & $4,736(27.2)$ & 6,471 & 190 & $1.40(1.17-1.68)$ & & $1.15(0.95-1.40)$ & \\
\hline & Mozambique & $1,777(10.2)$ & 2,499 & 40 & $0.84(0.60-1.18)$ & & $0.79(0.57-1.11)$ & \\
\hline & Other & $917(5.3)$ & 1,327 & 27 & $1.06(0.71-1.58)$ & & $0.82(0.54-1.24)$ & \\
\hline \multirow[t]{4}{*}{ Years in workforce ${ }^{c}$} & $0-9$ & 4,683 (26.9) & 6,391 & 85 & 1 & $<0.001$ & & \\
\hline & 10-19 & 4,753 (27.3) & 6,856 & 147 & $1.68(1.28-2.20)$ & & & \\
\hline & $20-29$ & $5,511(31.7)$ & 7,608 & 217 & $2.11(1.64-2.71)$ & & & \\
\hline & $\geq 30$ & $2,446(14.1)$ & 3,107 & 90 & $2.12(1.57-2.86)$ & & & \\
\hline \multirow[t]{2}{*}{ Type of work } & Surface & 1,537 (8.8) & 2,165 & 30 & 1 & 0.002 & 1 & 0.002 \\
\hline & Underground & $15,836(91.2)$ & 21,762 & 508 & $1.71(1.18-2.48)$ & & $1.75(1.20-2.54)$ & \\
\hline \multirow[t]{2}{*}{ Type of housing } & Hostel & $10,239(58.7)$ & 14,378 & 364 & 1 & $<0.001$ & 1 & 0.004 \\
\hline & Other & 7,206 (41.3) & 9,657 & 177 & $0.67(0.55-0.80)$ & & $0.74(0.61-0.91)$ & \\
\hline \multirow[t]{2}{*}{ Previous TB } & No & $15,427(88.5)$ & 21,413 & 430 & 1 & $<0.001$ & 1 & $<0.001$ \\
\hline & Yes & $2,005(11.5)$ & 2,605 & 111 & $2.08(1.69-2.57)$ & & $1.89(1.52-2.35)$ & \\
\hline \multirow[t]{2}{*}{ Self-reported ART use } & No & $16,956(97.3)$ & 23,386 & 523 & 1 & 0.42 & 1 & 0.64 \\
\hline & Yes & $471(2.7)$ & 625 & 17 & $1.23(0.76-1.99)$ & & $0.89(0.54-1.46)$ & \\
\hline Number of & $1-2$ & $4,640(26.6)$ & 6,513 & 159 & 1 & 0.001 & 1 & $<0.001$ \\
\hline \multirow[t]{2}{*}{ IPT prescriptions d,e } & $3-5$ & 1,855 (10.6) & 2,557 & 56 & $0.86(0.64-1.17)$ & & $0.81(0.59-1.09)$ & \\
\hline & $6+$ & $10,951(62.8)$ & 14,967 & 326 & $0.79(0.65-0.97)$ & & $0.63(0.51-0.77)$ & \\
\hline
\end{tabular}

${ }^{a}$ Adjusted for cluster using a fixed effect

${ }^{\mathrm{b}} P$ value for departure from linearity 0.002

c $P$ value for departure from linearity 0.02

${ }^{\mathrm{d}} P$ value for departure from linearity 0.83

e $P$ value for linear trend 0.02

${ }^{f}$ On 17,332 participants, adjusted for all variables shown

aHR, Adjusted hazard ratio; ART, Antiretroviral therapy; Cl, Confidence interval; HR, Hazard ratio; IPT, Isoniazid preventive therapy; pyrs, Person-years;

TB, Tuberculosis

\section{Discussion}

This study, one of only few to specifically address the durability of IPT, analysed the largest study cohort of people on IPT in the ART era. The large number of endpoints allowed for more power than previous studies to examine the timing and risk factors of incident TB after IPT.

TB incidence increased soon after stopping IPT; within 6 months, the TB incidence rate had more than doubled and, within a year, it was within the range of the community rate in the control clusters of the study (2.95 per 100 pyrs; $95 \%$ CI, 2.47-3.52) [6]. The previously published estimated TB incidence rate after IPT in a subset of our study participants was in the same order of magnitude [6]. The trend in incidence rates over time was also comparable, although our much larger sample size allowed us to look at this in more detail by assessing follow-up time in shorter periods. Increasing rates after IPT discontinuation have also been reported in highprevalence settings in the pre-ART $[24,25]$ and ART era $[4,9]$. In a medium-incidence setting (Brazil), TB incidence rates among TST-positive HIV-positive persons remained low for a median of 4.8 years following the start of IPT [26, 27]. In addition to a lower force of infection, a higher likelihood of cure due to a lower bacillary burden in latent infection was proposed as an explanation for the absence of rebound in their setting compared to in high-prevalence settings.

Our study design does not allow conclusions about the underlying mechanism of TB after IPT. Reinfection as a mechanism for TB after IPT is very likely in this population with a high annual risk of $M$. tuberculosis infection 
Table 3 Risk factors stratified by time after intended end of isoniazid preventive therapy (intention-to-treat)

\begin{tabular}{|c|c|c|c|c|c|c|c|c|}
\hline \multirow{2}{*}{ Total } & & \multicolumn{3}{|c|}{ Year 0-1 } & \multicolumn{3}{|c|}{ Year 1+ } & \multirow[b]{2}{*}{$P$ value ${ }^{\mathrm{b}}$} \\
\hline & & pyrs & TB & 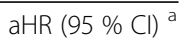 & pyrs & $\mathrm{TB}$ & $\overline{\mathrm{aHR}(95 \% \mathrm{Cl})^{\mathrm{a}}}$ & \\
\hline \multirow[t]{2}{*}{ Sex } & Male & 15,759 & 344 & 1 & 7,277 & 179 & 1 & 0.15 \\
\hline & Female & 709 & 15 & $0.82(0.49-1.38)$ & 290 & 3 & $0.35(0.11-1.11)$ & \\
\hline \multirow[t]{4}{*}{ Age, years } & $\leq 29$ & 2,229 & 18 & 1 & 992 & 12 & 1 & 0.54 \\
\hline & $30-39$ & 4,465 & 81 & $2.27(1.36-3.79)$ & 2,196 & 50 & $1.90(1.01-3.56)$ & \\
\hline & $40-49$ & 6,995 & 189 & $3.27(2.01-5.30)$ & 3,295 & 87 & $2.14(1.17-3.91)$ & \\
\hline & $\geq 50$ & 2,779 & 71 & $3.01(1.79-5.06)$ & 1,085 & 33 & $2.42(1.25-4.70)$ & \\
\hline \multirow[t]{4}{*}{ Years in workforce } & $0-9$ & 4,436 & 58 & 1 & 1,956 & 27 & 1 & 0.15 \\
\hline & 10-19 & 4,543 & 84 & $1.48(1.06-2.08)$ & 2,314 & 63 & $2.05(1.30-3.22)$ & \\
\hline & $20-29$ & 5,213 & 155 & $2.23(1.65-3.02)$ & 2,395 & 62 & $1.86(1.18-2.92)$ & \\
\hline & $\geq 30$ & 2,229 & 61 & $2.03(1.41-2.91)$ & 878 & 29 & $2.35(1.39-3.97)$ & \\
\hline \multirow[t]{2}{*}{ Type of work } & Surface & 1,465 & 20 & 1 & 700 & 10 & 1 & 0.98 \\
\hline & Underground & 14,932 & 339 & $1.71(1.09-2.69)$ & 6,830 & 169 & $1.72(0.91-3.27)$ & \\
\hline \multirow[t]{2}{*}{ Type of housing } & Hostel & 9,681 & 236 & 1 & 4,697 & 128 & 1 & 0.57 \\
\hline & Other & 6,787 & 123 & $0.69(0.55-0.86)$ & 2,870 & 54 & $0.62(0.45-0.85)$ & \\
\hline \multirow[t]{2}{*}{ Previous TB } & No & 14,618 & 281 & 1 & 6,795 & 149 & 1 & 0.63 \\
\hline & Yes & 1,838 & 78 & $2.15(1.68-2.77)$ & 767 & 33 & $1.93(1.32-2.81)$ & \\
\hline \multirow[t]{2}{*}{ Self-reported ART use } & No & 16,013 & 346 & 1 & 7,373 & 177 & 1 & 0.82 \\
\hline & Yes & 439 & 12 & $1.28(0.72-2.27)$ & 186 & 5 & $1.13(0.46-2.75)$ & \\
\hline \multirow[t]{3}{*}{ Number of IPT prescriptions } & $1-2$ & 4,321 & 99 & 1 & 2,192 & 60 & 1 & 0.86 \\
\hline & $3-5$ & 1,715 & 36 & $0.88(0.60-1.29)$ & 842 & 20 & $0.84(0.50-1.39)$ & \\
\hline & $6+$ & 10,433 & 224 & $0.82(0.65-1.05)$ & 4,534 & 102 & $0.79(0.42-1.46)$ & \\
\hline
\end{tabular}

${ }^{a}$ Adjusted for cluster. ${ }^{b}$ Test for interaction for factor with time

aHR, Adjusted hazard ratio; ART, Antiretroviral therapy; Cl, Confidence interval; HR, Hazard ratio; IPT, Isoniazid preventive therapy; pyrs, Person-years;

TB, Tuberculosis

(estimated at $20 \%$ [22]). TB contact studies in resourcelimited settings report the highest incidence of TB disease in the first year after acquisition [28], and therefore reinfection could certainly be responsible for some of the TB incidence in the first year after IPT. However, the rapid increase in incidence after stopping seems suggestive of a role for reactivation disease. Mathematical modelling of the rebound in TB incidence in three subSaharan African IPT trials in the pre-ART era estimated reactivation of persistent latent infection to be responsible for almost all TB after the end of IPT [29]. A recently published mathematical model of the Thibela study data also found that uncured latent infection among HIV-positive miners may partly explain the lack of population level impact [22].

Our crude estimate of the average incidence rate of TB disease in the first year after IPT that might be attributable due to reinfection [22] was lower than our observed estimate of the TB incidence rate in the first year after IPT. Despite the substantial variation in annual risk of infection, this was consistent across clusters. This supports our hypothesis that a proportion of the postIPT TB cases could be due to reactivation disease.
The rapid return of the TB incidence rate to baseline levels followed by a stable rate over time could therefore reflect a composite of both mechanisms: reactivation disease gradually replaced by reinfection disease, eventually reaching a steady state of a mixture of the two mechanisms. A similar time pattern in the mechanisms underlying recurrent $\mathrm{TB}$ disease after $\mathrm{TB}$ treatment was found in a molecular epidemiological study in South Africa, where early recurrences were primarily relapses and later recurrences were primarily reinfection [21]. Further, the episode start date was defined as the treatment start date, which is likely substantially later than the actual episode start date, and could have affected our estimate of the possible contribution of reinfection.

Across southern Africa a high proportion of recurrent TB disease is due to reinfection [18, 21, 30-32]. It has been argued that latent infection confers some protection against reinfection in high-prevalence settings [33]. It is unclear whether this protection could be removed by IPT, rendering the treated population at higher risk of reinfection. In our IPT cohort the TB incidence rate did not exceed that in the control arm, suggesting no increased risk of TB after IPT in our cohort. 
Our risk factor analysis showed that a longer duration of IPT led to lower rates of TB after IPT. There was no evidence for waning of this effect over time ( $\geq 12$ months compared to $<12$ months), although our analysis may have had too short a follow-up period or may have been underpowered to demonstrate this. An extended duration of IPT further reduced TB incidence among TSTand HIV-positive persons in high incidence settings [7]. However, this effect also waned over time after the end of IPT [9], suggesting that a longer duration of IPT does not increase the durability of protection in highprevalence settings. Mathematical modelling corroborates this and further estimates that TB disease among those treated with rifamycin-containing preventive therapy is less likely to be due to reactivation, based on a Ugandan study showing a slower rebound in participants having used a regimen including rifamycins compared to only isoniazid [25,29]. Rifamycins have a higher sterilizing potential; research into the use of regimens which include them is ongoing. Thus far, trials comparing regimens with isoniazid and rifamycin have not shown lower rates of TB in a high transmission setting, although most were not designed to show superiority [34, 35].

The risk factors for TB after IPT identified herein are well-known and the same as before IPT [36], suggesting IPT does not modify these. We found no evidence for different risk factors in the first year compared to later years after IPT. Follow-up might not have been long enough or our analysis might have been underpowered to identify a difference, however. The risk factors we identified mediate risks of reinfection as well as reactivation, thereby not helping to differentiate between the two underlying disease mechanisms.

The strengths of our analysis include the large number of TB diagnoses and person-years of follow-up in a wellcharacterised employment cohort resulting in higher power than other studies to date to test hypotheses, in particular relating to effect modification. Several important risk factors were not measured and could therefore not be adjusted for, most importantly silicosis and HIV status, which combine multiplicatively [36]. It would have been interesting to stratify the trajectory of $\mathrm{TB}$ rates after IPT by HIV status, as HIV-positive patients (especially those with a low CD4 count) might progress to disease faster after reinfection than HIV-negative patients. As the HIV prevalence among the gold miners is higher than among the general population, this might limit the direct comparability of our findings [37]. However, we applied the same HIV prevalence to arrive at our modelled estimate of TB incidence after reinfection, thereby not affecting our conclusions of the contribution of the underlying mechanism of disease.

We were not able to include ART use as a timeupdated variable, whereas we know that ART use increased during the course of the study [22] and is highly associated with decreasing risk of $\mathrm{TB}$ disease $[22,38]$. Our analysis only included self-reported ART usage, which likely represents misclassification; however, removing this variable from the multivariable analysis did not change the estimates. Selection bias may have arisen by a proportion of participants at higher risk of $\mathrm{TB}$ leaving the workforce (during or following IPT). Follow-up practices differed during IPT and after IPT, possibly causing ascertainment bias leading to an underestimate of $\mathrm{TB}$ incidence after IPT. The analyses did not take account of possible incomplete adherence to IPT, possibly leading to an overestimate of IPT duration and therefore of TB incidence rates during IPT. This potential misclassification would only occur in the AT analysis, thereby not impacting our main outcome and conclusions. Finally, we did not have access to cluster-specific estimates of HIV prevalence, case detection and pre-treatment loss to follow-up. This might have led to an over- or underestimate of the cluster-specific $\mathrm{TB}$ incidence rates as calculated by our model.

\section{Conclusions}

The durability of protection by IPT was lost within 6-12 months in this population with a high annual risk of $M$. tuberculosis infection and a high HIV prevalence. The observed TB incidence in the first year after IPT was higher than the crude estimate of the TB incidence attributable to reinfection, suggesting that reactivation of persistent latent infection played a role in the rapid return to baseline TB incidence. Further studies of the durability of alternative (rifamycin-containing) TB preventive therapy regimens, alone or in combination with ART, are warranted.

\section{Additional file}

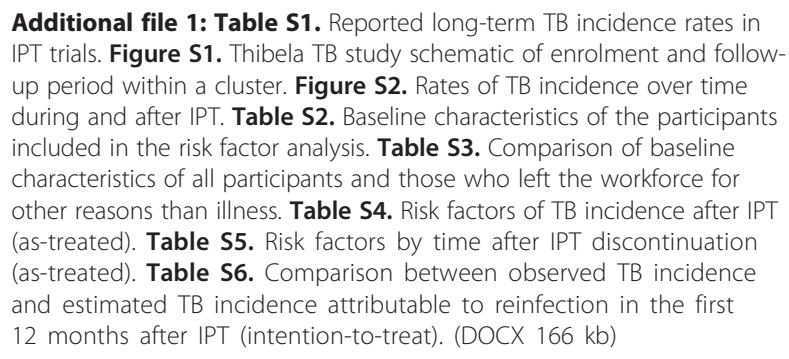

Competing interests

The authors declare that they have no competing interests.

Authors' contributions

$\mathrm{SH}$ and $\mathrm{KF}$ conceived and designed the experiments and analysed the data. EV developed the mathematical model and calculated the estimated rates. $A G, V C, J L, G C$ and KF were involved in collecting the data. All authors 
interpreted the data and wrote the manuscript. SH wrote the first draft. All authors read and approved the final manuscript.

\section{Acknowledgements}

The Thibela TB study was funded through the Consortium to Respond Effectively to the AIDS TB Epidemic (CREATE), via a grant from the Bill \& Melinda Gates Foundation (19790.01). SH was supported by the Swiss National Science Foundation (Fellowship for prospective researchers, PBSKP3_145774) and the European Union (Marie Curie International Outgoing Fellowship for Career Development PIOF-GA-2012-332311 to SH). AG was supported by a Public Health Career Scientist award from the UK Department of Health. VC, JL, EV, GC and KF were funded by a grant from CREATE (see above). KF also received support from the MRC and DFID (MR/K012126/1)

\section{Author details}

'TB Centre, London School of Hygiene \& Tropical Medicine, London, UK. ${ }^{2}$ Department of Global Health, Academic Medical Center, University of Amsterdam, Amsterdam Institute for Global Health and Development, Amsterdam, The Netherlands. ${ }^{3}$ Desmond Tutu HIV Centre, Institute for Infectious Diseases and Molecular Medicine, University of Cape Town, Cape Town, South Africa. ${ }^{4}$ Department of Internal Medicine, School of Medicine, Makerere University College of Health Sciences, Kampala, Uganda. ${ }^{5}$ The School of Public Health, University of the Witwatersrand, Johannesburg, South Africa. ${ }^{6}$ School of Nursing \& Public Health (Africa Centre for Population Health), University of KwaZulu-Natal, Durban, South Africa. ${ }^{7}$ Aurum Institute, Johannesburg, South Africa. ${ }^{8}$ Public Health England, London, UK. ${ }^{9}$ Advancing Care and Treatment for TB and HIV, MRC Collaborating Centre of Excellence, Johannesburg, South Africa.

Received: 14 December 2015 Accepted: 2 March 2016 Published online: 23 March 2016

\section{References}

1. World Health Organization. Report on global tuberculosis control. 2015. http://apps.who.int/iris/bitstream/10665/191102/1/9789241565059_eng. pdf?ua=1. Accessed 25 November 2015.

2. World Health Organization. Interim policy on collaborative TB/HIV activities. 2004. http://apps.who.int/iris/bitstream/10665/78705/1/WHO_HTM_TB_2004. 330_eng.pdf. Accessed 17 March 2009.

3. Akolo C, Adetifa I, Shepperd S, Volmink J. Treatment of latent tuberculosis infection in HIV infected persons. Cochrane Database Syst Rev. 2010;1: CD000171.

4. Rangaka M, Wilkinson RJ, Boulle A, Glynn JR, Fielding K, Van Cutsem G, et al. Isoniazid plus antiretroviral therapy to prevent tuberculosis: a randomised double-blind placebo-controlled trial. Lancet. 2014;384(9944):682-90.

5. Kirenga BJ, Worodria W, Massinga-Loembe M, Nalwoga T, Manabe YC, Kestens $L$, et al. Tuberculin skin test conversion among HIV patients on antiretroviral therapy in Uganda. Int J Tuberc Lung Dis. 2013;17(3):336-41.

6. Churchyard GJ, Fielding KL, Lewis JJ, Coetzee L, Corbett EL, Godfrey-Faussett $P$, et al. A trial of mass isoniazid preventive therapy for tuberculosis control. N Engl J Med. 2014;370(4):301-10.

7. Samandari T, Agizew TB, Nyirenda S, Tedla Z, Sibanda T, Shang N, et al. 6month versus 36-month isoniazid preventive treatment for tuberculosis in adults with HIV infection in Botswana: a randomised, double-blind, placebocontrolled trial. Lancet. 2011;377(9777):1588-98.

8. Martinson NA, Barnes GL, Moulton LH, Msandiwa R, Hausler H, Ram M, et al. New regimens to prevent tuberculosis in adults with HIV infection. N Engl J Med. 2011;365(1):11-20.

9. Samandari T, Agizew TB, Nyirenda S, Tedla Z, Sibanda T, Mosimaneotsile B, et al. Tuberculosis incidence after 36 months' isoniazid prophylaxis in HIVinfected adults in Botswana: a posttrial observational analysis. AIDS. 2015;29(3):351-9.

10. Enarson DA, Grzybowski S. Incidence of active tuberculosis in the native population of Canada. Can Med Assoc J. 1986;134(10):1149-52.

11. Comstock GW, Baum C, Snider Jr DE. Isoniazid prophylaxis among Alaskan Eskimos: a final report of the bethel isoniazid studies. Am Rev Respir Dis. 1979;119(5):827-30.

12. Fielding KL, Grant AD, Hayes RJ, Chaisson RE, Corbett EL, Churchyard GJ. Thibela TB: design and methods of a cluster randomised trial of the effect of community-wide isoniazid preventive therapy on tuberculosis amongst gold miners in South Africa. Contemp Clin Trials. 2011;32(3):382-92.

13. Grant AD, Coetzee L, Fielding KL, Lewis JJ, Ntshele S, Luttig MM, et al. 'Team up against TB': promoting involvement in Thibela TB, a trial of communitywide tuberculosis preventive therapy. AIDS. 2010;24 Suppl 5:S37-44.

14. Hanifa Y, Grant AD, Lewis J, Corbett EL, Fielding K, Churchyard G. Prevalence of latent tuberculosis infection among gold miners in South Africa. Int J Tuberc Lung Dis. 2009;13(1):39-46.

15. Lewis JJ, Fielding KL, Grant AD, Chihota VN, Popane F, Luttig M, et al. Eligibility for isoniazid preventive therapy in South African gold mines. PLoS One. 2013;8(11):e81376.

16. Lewis JJ, Charalambous S, Day JH, Fielding KL, Grant AD, Hayes RJ, et al. HIV infection does not affect active case finding of tuberculosis in South African gold miners. Am J Respir Crit Care Med. 2009;180(12):1271-8.

17. Smieja MJ, Marchetti CA, Cook DJ, Smaill FM. Isoniazid for preventing tuberculosis in non-HIV infected persons. Cochrane Database Syst Rev. 2000;2:CD001363.

18. Verver S, Warren RM, Beyers N, Richardson M, van der Spuy GD, Borgdorff MW, et al. Rate of reinfection tuberculosis after successful treatment is higher than rate of new tuberculosis. Am J Respir Crit Care Med. 2005:171(12):1430-5.

19. Lahey T, Mackenzie T, Arbeit RD, Bakari M, Mtei L, Matee M, et al. Recurrent tuberculosis risk among HIV-infected adults in Tanzania with prior active tuberculosis. Clin Infect Dis. 2013;56(1):151-8.

20. Hayes RJ, Moulton LH. Cluster randomised trials. Interdisciplinary Statistics Series. London: Chapman \& Hall/CRC Press; 2009.

21. Marx FM, Dunbar R, Enarson DA, Williams BG, Warren RM, van der Spuy GD, et al. The temporal dynamics of relapse and reinfection tuberculosis after successful treatment: a retrospective cohort study. Clin Infect Dis. 2014:58(12):1676-83.

22. Vynnycky E, Sumner T, Fielding KL, Lewis JJ, Cox AP, Hayes RJ, et al. Tuberculosis control in South African gold mines: mathematical modeling of a trial of community-wide isoniazid preventive therapy. Am J Epidemiol. 2015;181(8):619-32.

23. Kleinschmidt I, Churchyard G. Variation in incidences of tuberculosis in subgroups of South African gold miners. Occup Environ Med. 1997;54(9):636-41.

24. Quigley MA, Mwinga A, Hosp M, Lisse I, Fuchs D, Porter JDH, et al. Long-term effect of preventive therapy for tuberculosis in a cohort of HIV-infected Zambian adults. AIDS. 2001;15(2):215-22.

25. Johnson JL, Okwera A, Hom DL, Mayanja H, Mutuluuza Kityo C, Nsubuga P, et al. Duration of efficacy of treatment of latent tuberculosis infection in HIV-infected adults. AIDS. 2001;15(16):2137-47.

26. Golub JE, Saraceni V, Cavalcante SC, Cohn S, Pacheco AG, Lau B, et al. Durability of isoniazid preventive therapy in HIV-positive patients in a medium burden TB setting. Atlanta, GA: 20th Conference on Retroviruses and Opportunistic Infections; 2012. Poster 190 LB.

27. Golub JE, Cohn S, Saraceni V, Cavalcante SC, Pacheco AG, Moulton LH, et al. Long-term protection from isoniazid preventive therapy for tuberculosis in HIV-infected patients in a medium-burden tuberculosis setting: The TB/HIV in Rio (THRio) Study. Clin Infect Dis. 2015;60(4):639-45.

28. Fox GJ, Barry SE, Britton WJ, Marks GB. Contact investigation for tuberculosis: a systematic review and meta-analysis. Eur Respir J. 2013;41(1):140-56.

29. Houben RM, Sumner T, Grant AD, White RG. Ability of preventive therapy to cure latent Mycobacterium tuberculosis infection in HIVinfected individuals in high-burden settings. Proc Natl Acad Sci U S A. 2014;111(14):5325-30

30. van Rie A, Warren R, Richardson M, Victor TC, Gie RP, Enarson DA, et al. Exogenous reinfection as a cause of recurrent tuberculosis after curative treatment. N Engl J Med. 1999:341(16):1174-9.

31. Charalambous S, Grant AD, Moloi V, Warren R, Day JH, van Helden P, et al. Contribution of reinfection to recurrent tuberculosis in South African gold miners. Int J Tuberc Lung Dis. 2008;12(8):942-8.

32. Crampin AC, Mwaungulu JN, Mwaungulu FD, Mwafulirwa DT, Munthali K, Floyd S, et al. Recurrent TB: relapse or reinfection? The effect of HIV in a general population cohort in Malawi. AIDS. 2010;24(3):417-26.

33. Andrews JR, Noubary F, Walensky RP, Cerda R, Losina E, Horsburgh CR. Risk of progression to active tuberculosis following reinfection with Mycobacterium tuberculosis. Clin Infect Dis. 2012;54(6):784-91.

34. Sharma SK, Sharma A, Kadhiravan T, Tharyan P. Rifamycins (rifampicin, rifabutin and rifapentine) compared to isoniazid for preventing tuberculosis 
in HIV-negative people at risk of active TB. Cochrane Database Syst Rev. 2013;7:CD007545.

35. Stagg HR, Zenner D, Harris RJ, Munoz L, Lipman MC, Abubakar I. Treatment of latent tuberculosis infection: a network meta-analysis. Ann Intern Med. 2014;161(6):419-28.

36. Corbett EL, Churchyard GJ, Clayton TC, Williams BG, Mulder D, Hayes RJ, et al. HIV infection and silicosis: the impact of two potent risk factors on the incidence of mycobacterial disease in South African miners. AIDS. 2000;14(17):2759-68.

37. Shisana O, Rehle T, Simbayi L, Zuma K, Jooste S, Zungu N, et al. South African National HIV Prevalence, Incidence and Behaviour Survey. 2012. http://www.hsrc.ac.za/en/research-data/view/6871. Accessed 2 December 2013.

38. Suthar AB, Lawn SD, del Amo J, Getahun H, Dye C, Sculier D, et al. Antiretroviral therapy for prevention of tuberculosis in adults with HIV: a systematic review and meta-analysis. PLoS Med. 2012;9(7):e1001270.

Submit your next manuscript to BioMed Central and we will help you at every step:

- We accept pre-submission inquiries

- Our selector tool helps you to find the most relevant journal

- We provide round the clock customer support

- Convenient online submission

- Thorough peer review

- Inclusion in PubMed and all major indexing services

- Maximum visibility for your research

Submit your manuscript at www.biomedcentral.com/submit
Biomed Central 